\title{
Does Doppler myocardial imaging give new insights or simply old information revisited?
}

Current standard cardiac ultrasound techniques derive their information on myocardial function indirectly either from variables measured from grey scale endocardial and epicardial specular reflections or from blood pool Doppler indices. Doppler myocardial imaging (DMI) is a new cardiac ultrasound imaging technique in which the Doppler principle has been applied to measure a number of indices of myocardial function. ${ }^{1-3}$ This technique is based on colour Doppler principles and has the potential to quantify regional intramural velocities by detecting consecutive phase-shifts of the ultrasound echoes from contracting myocardium. There are several advantages of this technique over standard grey scale $M$ mode and cross sectional imaging. The velocity of motion at a large number of neighbouring intramyocardial sites can be determined and distinguished very rapidly. The Doppler technique measures frequency shift rather than signal amplitude, and the image information is not greatly affected by tissue attenuation. Thus it is possible to obtain diagnostic quality DMI images from patients who would be considered poorly echogenic as a result of chest wall attenuation effects on standard grey scale imaging. ${ }^{4}$

The technology changes involved in obtaining DMI velocity images are described by Erbel et al an accompanying editorial on page 193 . From mean velocity information, regional myocardial accelerations can be calculated and displayed in a two-dimensional format. In addition, reflected Doppler energy can be measured. This variable directly measures tissue backscatter intensity and is derived by determining the power of the reflected Doppler signal. Doppler energy information is both velocity and angle independent. In terms of blood pool imaging, it has been shown to be directly equivalent to integrated backscatter data. ${ }^{5}$

In vitro phantom studies have confirmed the accuracy of DMI velocity encoding over the range of velocities at which normal and abnormal myocardium moves. ${ }^{6}$ Velocity estimation has been shown to be affected by target velocity, target material, system receive gain, and the pulse train size, but the inherent error is at worst $\pm 10 \%$ of the true mean velocity. ${ }^{6}$ The spatial resolution of both the two-dimensional velocity and energy maps is at best $1 \times$ $1 \mathrm{~mm} .^{2}$

\section{DMI studies of normal myocardial function}

Regional intramural velocities, acceleration, and reflected Doppler energy can be displayed as a two-dimensional image with frame rates similar to grey-scale imaging. Intramural velocities and energy can also be displayed in an $M$ mode format. $M$ mode velocity studies in normal patients have confirmed the presence of reproducible transmural velocity gradients across the septum and left and right ventricular free walls during the cardiac cycle. DMI $M$ mode velocity studies have confirmed the normal range of velocity distribution and timing of peak velocity gradients within the left ventricular posterior wall and interventricular septum. ${ }^{7} \mathrm{DMI} \mathrm{M}$ mode studies have also been used to interrogate regional long-axis shortening of the heart and have demonstrated a base-apex longitudinal velocity gradient in both the septum and ventricular free walls with the highest long-axis shortening velocities recorded at the cardiac base and with almost zero velocities recorded at the apex. Thus the DMI technique has the potential to characterise regional long-axis function of the heart including regional abnormalities in long-axis shortening and relaxation. Such information is currently not available from standard grey-scale imaging. DMI velocities have also been shown to change in a predictable manner in response to both positive and negative inotropes in an animal model. In addition, pulsed Doppler DMI velocity sampling of the myocardium can determine regional myocardial peak velocities and their temporal relation to mechanical and electrical events in the heart.

Left ventricular diastolic and left atrial function Earlier ultrasound indices of diastolic function have been derived either from digitised $M$ mode traces of the left ventricular endocardial surfaces or from Doppler indices derived from blood velocities measured in the left heart. In contrast, DMI provides a direct measure of intramural velocities during left ventricular relaxation. Preliminary studies have shown that DMI indices of myocardial relaxation parallel transmitral Doppler blood flow measurements and normal changes in blood flow associated with ageing. ${ }^{8}$ Others have shown that DMI derived diastolic velocities of long axis lengthening correlate with normal and abnormal atrioventricular valve ring velocities during diastole. ${ }^{9}$ In addition, DMI indices have been shown to correlate well with both left atrial wall function indices and indices of left atrial appendage function. ${ }^{10}$ However, the extent to which these new indices provide new clinical information remains to be determined.

\section{DMI studies in cardiomyopathies}

Clinical studies in the dilated cardiomyopathies (DCM), hypertrophic cardiomyopathies (HCM), and patients with concentric left ventricular hypertrophy have determined a range of abnormalities in the distribution of intramural velocities that cannot be demonstrated by either standard grey-scale two-dimensional or $\mathrm{M}$ mode techniques. These studies have shown a significant decrease in mean velocities and velocity gradients during all systole phases during atrial contraction in DCM patients. ${ }^{11}{ }^{12}$ In HCM patients, velocity gradients were significantly decreased or reversed in all systolic cardiac phases despite apparently normal $M$ mode fractional thickening indices. ${ }^{13}$ These abnormalities are likely to be caused by the abnormal myocardial architecture in HCM patients and may prove to be a new ultrasound marker specific for this condition.

DMI studies in ischaemic heart disease

DMI can determine regional transmural acute and 
MAPS
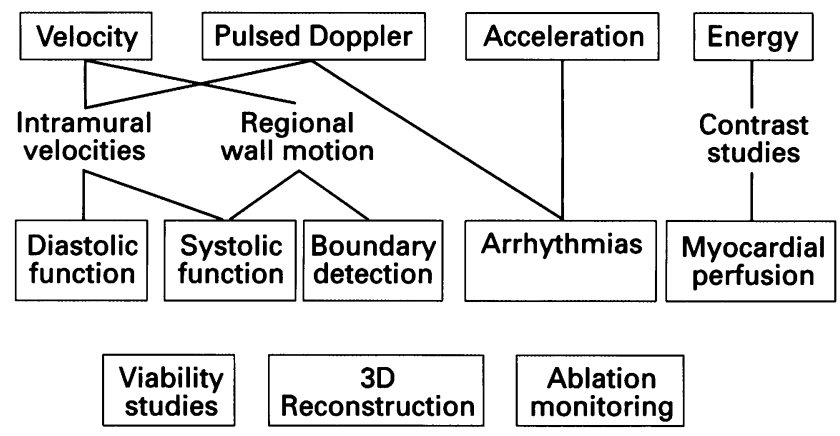

The potential clinical applications of Doppler myocardial imaging.

chronic changes in myocardial contractile function induced by ischaemia. ${ }^{12-14}$ These have been determined both in animal models and in patients. The earliest changes induced by ischaemia have been recorded using the DMI pulsed Doppler technique to identify changes in regional velocity. Significant early changes in regional diastolic velocities in the ischaemic zone were found after only 15 seconds ischaemia in a dog model. ${ }^{15}$ Later changes in systolic peak velocities were also observed which paralleled changes in the transmural velocity gradient obtained by two-dimensional DMI reduction in wall thickening on $M$ mode grey-scale imaging. Encouraging results have also been reported with regard to reversible ischaemic changes detected using the DMI single sample volume pulsed Doppler technique in conjunction with low-dose dobutamine infusions. ${ }^{16}$ Predictable changes in transmural velocities associated with a marked reduction in Doppler energy signal strength have also been noted in patients with acute myocardial infarction. ${ }^{14}$

Doppler energy evaluation of myocardial perfusion Doppler energy has been shown to be effective in detecting the presence of left heart contrast agents within the myocardium in both a closed-chest animal model and in normal subjects. ${ }^{2}$ Cross sectional Doppler energy imaging has also been shown to detect regional perfusion abnormalities, perfusion changes caused by concomitant dipyridamole infusion, and myocardial reperfusion in an animal model. ${ }^{17}$ What role any of the Doppler energy techniques might play in a clinically effective ultrasound approach remains to be determined but currently DMI shows as much promise as either radiofrequency data or second harmonic imaging.

\section{DMI evaluation of arrhythmias}

The combination of DMI velocity and acceleration mapping using two-dimensional, $M$ mode, and pulsed DMI techniques can identify specific normal and abnormal sequences of myocardial acceleration which accurately reflect the mode and timing of electrical depolarisation. Abnormal early regional changes in velocity or acceleration associated with either Wolff-Parkinson-White bypass tracts, unifocal ventricular extrasystoles, or sustained ventricular tachycardias have been accurately located, as has the immediate normalisation in depolarisation induced during the monitoring of radiofrequency ablation..$^{18} 19$

\section{Doppler myocardial imaging: advantages for} transthoracic 3-D reconstruction

Three-dimensional echocardiography (3-D) has been vali- dated as an accurate technique for determining left ventricular volume (LVV). However, the major limitation of transthoracic 3-D data acquisition is often the poor standard of grey-scale image quality available for reconstruction. In contrast, DMI is $(a)$ relatively independent of the amplitude of the ultrasonic signal returning from the interrogated myocardium and is less affected by the attenuating effect of the chest wall and (b) the DMI algorithm is a powerful boundary detection technique, and hence potentially is able to provide a more complete data set for 3-D reconstruction than that obtained by grey-scale imaging. ${ }^{20} \mathrm{LVV}$ measurements have been validated by comparing transthoracic 3-D DMI with standard grey-scale imaging techniques. ${ }^{21}$ Such studies have shown that, despite the slightly inferior spatial resolution of DMI, 3-D LVV estimation was equally well effected by both. However, the superior boundary detection and relative lack of chest wall signal attenuation inherent in DMI allowed for accurate volume measurements and successful 3-D volume reconstruction in difficult-to-image patients in whom grey-scale imaging failed to acquire a data-set. ${ }^{21}$

\section{Doppler imaging of skeletal muscle and skeletal muscle assist devices}

Potential applications of DMI are not confined to cardiac imaging. Early validation work has demonstrated that DMI could be used to identify contracting skeletal muscle groups. ${ }^{1}$ Since then, skeletal muscle contraction in healthy volunteers has been systematically examined. ${ }^{22}$ This study demonstrated the capability of DMI to identify tetanic skeletal muscle contraction; to differentiate between active contraction and passive muscle movement; and to characterise isotonic, isometric, and reflex skeletal muscle contraction profiles. Thus there may be considerable potential for further evaluation of DMI in neurological and musculoskeletal applications. In particular, DMI may have practical application in the design and validation of biomechanical assist systems such as latissimus dorsi cardiomyoplasty. ${ }^{23} 24$

\section{GEORGE R SUTHERLAND ALEKSANDRA LANGE}

Department of Cardiology, PRZEMYSLAW PALKA

Western General Hospital,

Crewe Road,

Edinburgh EH4 $2 X U$

NEIL GRUBB

Department of Cardiology,

Royal Infirmary,

Edinburgh EH3 9 YW

ALAN FLEMING

W NORMAN MCDICKEN

Department of Medical Physics $\mathbb{E}$ Medical Engineering,

Royal Infirmary,

Edinburgh EH3 $9 Y W$

AL is a Scottish Office Home \& Health Department Research Fellow. PP is a Chest Heart \& Stroke Association Research Fellow.

1 McDicken WN, Sutherland GR, Moran CM, Gordon L. Colour Doppler velocity imaging of the myocardium. Ultrasound Med Biol 1992;18:651-4.

2 Sutherland GR, Steward MJ, Grounstroem KWE, Moran CM, Fleming $\mathrm{AD}$, Guell-Peris PJ, et al. Colour Doppler myocardial imaging: a new technique for the assessment of myocardial function. $7 \mathrm{Am}$ Soc Echocardiogr 1994;7:441-58.

3 Miyatake $K$, Yamagishi $M$, Tanaka $N$, Uematsu $M$, Yamazaki $N$, Yoshitake $M$, et al. A new method for the evaluation of left ventricular wall motion by color-coded tissue Dopple

4 Palka P, Lange A, Fleming AD, Sutherland GR, Fenn LN, McDicken WN. Doppler tissue imaging: myocardial wall motion velocities in normal subjects. F Am Soc Echocardiogr 1995;8,659-68.

5 Schwarz KQ, Bezante GP, Chen X. When can Doppler be used in place of integrated backscatter as a measure of scattered ultrasound intensity? intrasound in Medicine $\mathcal{E}$ Biology 1995;21:231-42.

6 Fleming AD, McDicken WN, Sutherland GR, Hoskins PR. Assessment of colour Doppler tissue imaging using test phantoms. Ultrasound in colour Doppler tissue imaging using

7 Fleming AD, Xia X, McDicken WN, Sutherland GR, Fenn L. Myocardial 
velocity gradient by Doppler imaging. Br $\mathcal{F}$ Radiol 1994;67:679-88.

8 Palka P, Fleming AD, Lange A, Fenn LN, Sutherland GR, McDicken WN. Age-related transmural peak mean velocities and peak velocity gra-
dients by Doppler myocardial imaging in normal subjects. Eur Heart $\mathcal{F}$ 1996;17:940-50.

9 Garcia MJ, Rodriguez L, Ares M, Griffin BP, Thomas JD, Klein AL Differentiation of constrictive pericarditis from restrictive cardiomyopathy: assessment of left ventricular diastolic velocities in longitudinal axis by Doppler tissue imaging. 7 Am Coll Cardiol 1996;27:108-14.

10 Rodriguez L, Nakatani S, Leung D, Griffin B, Stewart W, Thomas JD, et al. odriguez L, Nakatani S, Leung D, Griffin B, Stewart W, Thomas JD, et al. Measurement of atrial appendage cycle length by transthoracic echocardiography using Doppler tissue imaging (abstract). f Am Soc Echocardiogr

11 Lange A, Palka P, Sutherland GR, Fleming AD, Fenn LN, Bouki KP, et al. Doppler tissue imaging assessment of systolic and diastolic transmural velocity gradients in dilated cardiomyopathy: a new diagnostic index (abstract). Eur Heart f 1995;16(suppl):298.

12 Uematsu M, Miyatake K, Tanaka N, Matsuda H, Sano A, Yamazaki N, et al. Myocardial velocity gradient as a new indicator of regional left ventricular contraction: detection by a two-dimensional tissue doppler imaging technique. $\mathcal{F}$ Am Coll Cardiol 1995;26:217-23.

13 Palka P, Lange A, Sutherland GR, Fleming AD, Fenn LN, Bouki K, et al. Doppler tissue imaging: assessment of systolic and diastolic transmura velocity gradients in hypertrophic cardiomyoapthy. A new diagnostic index (abstract). Eur Heart $\mathcal{f} 1995 ; 16$ (suppl): 105 .

14 Stewart MJ, Sutherland GR, Moran CN, Fleming AD, Fenn LN, McDicken WN. Imaging of ischaemic and infarcted myocardium by Doppler tissue imaging (abstract), Circulation 1993;88:I-47.

15 Garcia-Fernandez MA, Azevedo J, Puerta M, Moreno E, Torrecilla E, San Roman D. Quantatative analysis of segmental left ventricular wall dysfunction by pulsed doppler tissue imaging. $A$ new insight into diastolic performance (abstract). Eur Heart $\mathcal{f}$ 1995; 16(suppl):451.
16 von Bibra H, Tuchnitz A, Firschke C, Schühlen H, Schömig A. Doppler tissue imaging of left ventricular myocardium-initial results during pharmacologic stress (abstract). I Am Coll Cardiol 1995; P57A.

17 Sutherland GR, von Bibra H, Tuchnitz A, Henke J, Schönig A Transthoracic detection of regional myocardial perfusion abnormalities using a pervenous contrast agent-a comparative study of Doppler energy and grey scale imaging. Br Heart $\mathcal{f} 1995 ; 73$ suppl 3:57.

18 Yamagishi M, Tanaka N, Itoh S, Miyatake K, Yamayaki N, Hirama M. An enhanced method for detection of early contraction site of ventricles in Wolff-Parkinson-White syndrome using color coded tissue Doppler echocardiography (abstract). $\mathcal{F}$ Am Soc Echocardiogr 1993;6:30.

19 Sutherland GR, Pons Llado GP, Carreras F, Vinolas X, Oter R, Subiren $M$, et al. Doppler myocardial imaging in the evaluation of normal and abnormal ventricular depolarisation. $\mathrm{Br}$ Heart $\mathcal{f} 1995 ; 73$ suppl 3:85.

20 Sutherland GR, Caso P, Palka P, Fenn LN, Lange A, McDicken WN. Doppler myocardial imaging assessment of left ventricular volume and area: a comparison with grey-scale imaging (abstract). Eur Heart $\mathcal{f}$ 1995;16(suppl):392.

21 Lange A, Bouki K, Fenn LN, Palka P, McDicken WN, Sutherland WN. A comparison study of grey scale versus Doppler tissue imaging left ventricular volume measurement using three dimensional reconstruction (abstract). Eur Heart $\mathcal{f}$ 1995;16(suppl):266.

22 Grubb NR, Fleming A, Fox KAA, Sutherland GR. Evaluation of Doppler tissue imaging for the assessment of skeletal muscle function in healthy volunteers. Radiology 1995;194:837-42.

23 Grubb NR, Sutherland GR, Campanella C. Optimisation of myostimulation in dynamic cardiomyoplasty. Eur 7 Cardiothorac Surg 1995;9:45-9.

24 Grubb NR, Sutherland GR, Campanella C, Fleming A, Fox KAA Evaluation of Doppler tissue imaging for non-invasive assessment of latissimus dorsi function and ventricular wall motion after dynamic cardiomyoplasty. Radiology 1996;199:59-64. 